DOI: 10.2478/ausm-2014-0010

\title{
Related fixed point theorem for four complete metric spaces
}

\author{
Vishal Gupta \\ Maharishi Markandeshwar University \\ Department of Mathematics \\ Mullana, Ambala, Haryana (India) \\ email: vishal.gmn@gmail.com
}

\begin{abstract}
In the present paper, we obtain a new result on fixed point theorem for four metric spaces. Here we choose continuous mappings. In fact our result is the generalization of many results of fixed point theorem on two and three metric spaces. We also give some illustrative examples to justify our result.
\end{abstract}

\section{Introduction}

Related fixed point theorems on two metric spaces have been studied by B. Fisher [2]. Also some fixed point theorems on three metric spaces have been studied by B. Fisher et al [3], R. K. Jain et al. [4], R. K. Namdeo and B. Fisher [7], K. Kikina et al. [6], Z. Ansari et al. [1], and V. Gupta [8]. Also, the fixed point theorems on four metric spaces have been studied by L. Kikina et al. [5]. In the present paper a generalization is given for four complete metric space. Our theorem improves Theorem (2.1) of R. K. Jain et al. [4].

The following fixed point theorem was proved by R. K. Jain, H. K. Sahu, B. Fisher [4].

Theorem 1 Let $(\mathrm{X}, \mathrm{d}),(\mathrm{Y}, \rho)$ and $(\mathrm{Z}, \sigma)$ be complete metric spaces. If $\mathrm{T}$ is continuous mapping of $\mathrm{X} \mapsto \mathrm{Y}, \mathrm{S}$ is a continuous mapping of $\mathrm{Y} \mapsto \mathrm{Z}$ and $\mathrm{R}$ is mapping of $\mathrm{Z} \mapsto \mathrm{X}$ satisfying the inequalities

2010 Mathematics Subject Classification: 54H25, 47H10

Key words and phrases: complete metric spaces, fixed points, Cauchy sequences, continuous mappings 
$d\left(R S T x, R S T x^{\prime}\right) \leqslant c \max \left\{d\left(x, x^{\prime}\right), d(x, R S T x)\right.$,

$$
\left.d\left(x^{\prime}, R S T x^{\prime}\right), \rho\left(T x, T x^{\prime}\right), \sigma\left(S T x, S T x^{\prime}\right)\right\},
$$

$\rho($ TRSy, TRSy' $) \leqslant c \max \left\{\rho\left(y, y^{\prime}\right), \rho(y, T R S y)\right.$, $\left.\rho\left(y^{\prime}, T R S y^{\prime}\right), \sigma\left(S y, S y^{\prime}\right), d\left(R S y, R S y^{\prime}\right)\right\}$,

$\sigma\left(\operatorname{STR} z, \operatorname{STRz} z^{\prime}\right) \leqslant c \max \left\{\sigma\left(z, z^{\prime}\right), \sigma(z, \operatorname{STR} z)\right.$

$$
\left.\sigma\left(z^{\prime}, S T R z^{\prime}\right), d\left(R z, R z^{\prime}\right), \rho\left(T R z, T R z^{\prime}\right)\right\},
$$

$\forall x, x^{\prime} \in X, y, y^{\prime} \in Y$ and $z, z^{\prime} \in Z$, where $0 \leqslant c<1$, then RST has a unique fixed point $u \in X$, TRS has a unique fixed point $v \in Y$ and STR has a unique fixed point $w \in Z$. Further $\mathrm{Tu}=v, \mathrm{~S} v=w$ and $\mathrm{R} w=u$.

\section{Main result}

Theorem 2 Let $\left(\mathrm{Z}_{1}, \mathrm{~d}_{1}\right),\left(\mathrm{Z}_{2}, \mathrm{~d}_{2}\right),\left(\mathrm{Z}_{3}, \mathrm{~d}_{3}\right)$, and $\left(\mathrm{Z}_{4}, \mathrm{~d}_{4}\right)$ be complete metric spaces. If $A_{1}$ is a continuous mapping of $Z_{1} \mapsto Z_{2}, A_{2}$ is continuous mapping of $Z_{2} \mapsto Z_{3}, A_{3}$ is continuous mapping of $Z_{3} \mapsto Z_{4}$ and $A_{4}$ is a mappings of $\mathrm{Z}_{4} \mapsto \mathrm{Z}_{1}$, satisfying the inequalities

$$
\begin{aligned}
& d_{1}\left(A_{4} A_{3} A_{2} A_{1} z_{1}, A_{4} A_{3} A_{2} A_{1} z_{1}^{\prime}\right) \\
& \leqslant c \max \left\{d_{1}\left(z_{1}, z_{1}^{\prime}\right), d_{1}\left(z_{1}, A_{4} A_{3} A_{2} A_{1} z_{1}\right),\right. \\
& \quad d_{1}\left(z_{1}^{\prime}, A_{4} A_{3} A_{2} A_{1} z_{1}^{\prime}\right), d_{2}\left(A_{1} z_{1}, A_{1} z^{\prime}\right) \\
& \left.\quad d_{3}\left(A_{2} A_{1} z_{1}, A_{2} A_{1} z_{1}^{\prime}\right), d_{4}\left(A_{3} A_{2} A_{1} z_{1}, A_{3} A_{2} A_{1} z_{1}^{\prime}\right)\right\}, \\
& d_{2}\left(A_{1} A_{4} A_{3} A_{2} z_{2}, A_{1} A_{4} A_{3} A_{2} z_{2}^{\prime}\right) \\
& \leqslant c \max \left\{d_{2}\left(z_{2}, z_{2}^{\prime}\right), d_{2}\left(z_{2}, A_{1} A_{4} A_{3} A_{2} z_{2}\right),\right. \\
& \quad d_{2}\left(z_{2}^{\prime}, A_{1} A_{4} A_{3} A_{2} z_{2}^{\prime}\right), d_{3}\left(A_{2} z_{2}, A_{2} z_{2}^{\prime}\right), \\
& \left.\quad d_{4}\left(A_{3} A_{2} z_{2}, A_{3} A_{2} z_{2}^{\prime}\right), d_{1}\left(A_{4} A_{3} A_{2} z_{2}, A_{4} A_{3} A_{2} z_{2}^{\prime}\right)\right\}, \\
& d_{3}\left(A_{2} A_{1} A_{4} A_{3} z_{3}, A_{2} A_{1} A_{4} A_{3} z_{3}^{\prime}\right) \\
& \leqslant c \max _{3} d_{3}\left(z_{3}, z_{3}^{\prime}\right), d_{3}\left(z_{3}, A_{2} A_{1} A_{4} A_{3} z_{3}\right), \\
& \quad d_{3}\left(z_{3}^{\prime}, A_{2} A_{1} A_{4} A_{3} z_{3}^{\prime}\right), d_{4}\left(A_{3} z_{3}, A_{3} z_{3}^{\prime}\right), \\
& \left.\quad d_{1}\left(A_{4} A_{3} z_{3}, A_{4} A_{3} z_{3}^{\prime}\right), d_{2}\left(A_{1} A_{4} A_{3} z_{3}, A_{1} A_{4} A_{3} z_{3}^{\prime}\right)\right\}, \\
& d_{4}\left(A_{3} A_{2} A_{1} A_{4} z_{4}, A_{3} A_{2} A_{1} A_{4} z_{4}^{\prime}\right) \\
& \leqslant c_{4} \max _{2} d_{4}\left(z_{4}, z_{4}^{\prime}\right), d_{4}\left(z_{4}, A_{3} A_{2} A_{1} A_{4} z_{4}\right), \\
& \quad d_{4}\left(z_{4}^{\prime}, A_{3} A_{2} A_{1} A_{4} z_{4}^{\prime}\right), d_{1}\left(A_{4} z_{4}, A_{4} z_{4}^{\prime}\right), \\
& \left.d_{2}\left(A_{1} A_{4} z_{4}, A_{1} A_{4} z_{4}^{\prime}\right), d_{3}\left(A_{2} A_{1} A_{4} z_{4}, A_{2} A_{1} A_{4} z_{4}^{\prime}\right)\right\},
\end{aligned}
$$


$\forall z_{1}, z_{1}^{\prime} \in Z_{1}, z_{2}, z_{2}^{\prime} \in Z_{2}, z_{3}, z_{3}^{\prime} \in Z_{3}$ and $z_{4}, z_{4}^{\prime} \in Z_{4}$, where $0 \leqslant c<1$, then $A_{4} A_{3} A_{2} A_{1}$ has a unique fixed point $\alpha_{1} \in Z_{1}, A_{1} A_{4} A_{3} A_{2}$ has a unique fixed point $\alpha_{2} \in Z_{2}, A_{2} A_{1} A_{4} A_{3}$ has a unique fixed point $\alpha_{3} \in Z_{3}$ and $A_{3} A_{2} A_{1} A_{4}$ has a unique fixed point $\alpha_{4} \in Z_{4}$.

Further $A_{1} \alpha_{1}=\alpha_{2}, A_{2} \alpha_{2}=\alpha_{3}, A_{3} \alpha_{3}=\alpha_{4}, A_{4} \alpha_{4}=\alpha_{1}$.

Proof. Let $z_{1}^{0}$ be an arbitrary point in $Z_{1}$.

Define the sequence $\left\{z_{n}^{1}\right\},\left\{z_{n}^{2}\right\},\left\{z_{n}^{3}\right\}$ and $\left\{z_{n}^{4}\right\}$ in $Z_{1}, Z_{2}, Z_{3}$ and $Z_{4}$ respectively by

$$
\begin{aligned}
\left(A_{4} A_{3} A_{2} A_{1}\right)^{n} z_{1}^{0} & =z_{\mathfrak{n}}^{1} \\
A_{1} z_{n-1}^{1} & =z_{\mathfrak{n}}^{2} \\
A_{2} z_{\mathfrak{n}}^{2} & =z_{\mathfrak{n}}^{3} \\
A_{3} z_{\mathfrak{n}}^{3} & =z_{\mathfrak{n}}^{4} \\
A_{4} z_{\mathfrak{n}}^{4} & =z_{\mathfrak{n}}^{1} \quad \text { for } n=1,2, \ldots
\end{aligned}
$$

Applying inequality (5), we get,

$$
\begin{aligned}
& d_{2}\left(z_{n}^{2}, z_{n+1}^{2}\right)=d_{2}\left(A_{1} A_{4} A_{3} A_{2} z_{n-1}^{2}, A_{1} A_{4} A_{3} A_{2} z_{n}^{2}\right) \\
& \leqslant c \max \left\{\mathrm{d}_{2}\left(z_{n-1}^{2}, z_{n}^{2}\right), \mathrm{d}_{2}\left(z_{n-1}^{2}, A_{1} A_{4} A_{3} A_{2} z_{n-1}^{2}\right)\right. \text {, } \\
& \mathrm{d}_{2}\left(z_{\mathrm{n}}^{2}, A_{1} A_{4} A_{3} A_{2} z_{n}^{2}\right), \mathrm{d}_{3}\left(A_{2} z_{\mathrm{n}-1}^{2}, A_{2} z_{\mathrm{n}}^{2}\right) \text {, } \\
& \left.d_{4}\left(A_{3} A_{2} z_{n-1}^{2}, A_{3} A_{2} z_{n}^{2}\right), d_{1}\left(A_{4} A_{3} A_{2} z_{n-1}^{2}, A_{4} A_{3} A_{2} z_{n}^{2}\right)\right\} \\
& \mathrm{d}_{2}\left(z_{\mathrm{n}}^{2}, z_{\mathrm{n}+1}^{2}\right) \leqslant \mathrm{c} \max \left\{\mathrm{d}_{2}\left(z_{\mathrm{n}-1}^{2}, z_{\mathrm{n}}^{2}\right), \mathrm{d}_{2}\left(z_{\mathrm{n}-1}^{2}, z_{\mathrm{n}}^{2}\right), \mathrm{d}_{2}\left(z_{\mathrm{n}}^{2}, z_{\mathrm{n}+1}^{2}\right)\right. \text {, } \\
& \left.\mathrm{d}_{3}\left(z_{\mathrm{n}-1}^{3}, z_{\mathrm{n}}^{3}\right), \mathrm{d}_{4}\left(z_{\mathrm{n}-1}^{4}, z_{\mathrm{n}}^{4}\right), \mathrm{d}_{1}\left(z_{\mathrm{n}-1}^{1}, z_{\mathrm{n}}^{1}\right)\right\} \\
& \mathrm{d}_{2}\left(z_{\mathrm{n}}^{2}, z_{\mathrm{n}+1}^{2}\right) \leqslant c \max \left\{\mathrm{d}_{1}\left(z_{\mathrm{n}-1}^{1}, z_{\mathrm{n}}^{1}\right), \mathrm{d}_{2}\left(z_{\mathrm{n}-1}^{2}, z_{\mathrm{n}}^{2}\right), \mathrm{d}_{3}\left(z_{n-1}^{3}, z_{\mathrm{n}}^{3}\right), \mathrm{d}_{4}\left(z_{n-1}^{4}, z_{n}^{4}\right)\right\}
\end{aligned}
$$

Using inequality (6), we get,

$$
\begin{aligned}
\mathrm{d}_{3}\left(z_{\mathrm{n}}^{3}, z_{\mathrm{n}+1}^{3}\right)= & \mathrm{d}_{3}\left(A_{2} A_{1} A_{4} A_{3} z_{n-1}^{3}, A_{2} A_{1} A_{4} A_{3} z_{n}^{3}\right) \\
\leqslant & \max \left\{d_{3}\left(z_{n-1}^{3}, z_{n}^{3}\right), d_{3}\left(z_{n-1}^{3}, A_{2} A_{1} A_{4} A_{3} z_{n-1}^{3}\right),\right. \\
& d_{3}\left(z_{n}^{3}, A_{2} A_{1} A_{4} A_{3} z_{n}^{3}\right), d_{4}\left(A_{3} z_{n-1}^{3}, A_{3} z_{n}^{3}\right), \\
& \left.d_{1}\left(A_{4} A_{3} z_{n-1}^{3}, A_{4} A_{3} z_{n}^{3}\right), d_{2}\left(A_{1} A_{4} A_{3} z_{n-1}^{3}, A_{1} A_{4} A_{3} z_{n}^{3}\right)\right\} \\
d_{3}\left(z_{n}^{3}, z_{n+1}^{3}\right) \leqslant & c \max _{\{} d_{3}\left(z_{n-1}^{3}, z_{n}^{3}\right), d_{3}\left(z_{n-1}^{3}, z_{n}^{3}\right), d_{3}\left(z_{n}^{3}, z_{n+1}^{3}\right), \\
& \left.d_{4}\left(z_{n-1}^{4}, z_{n}^{4}\right), d_{1}\left(z_{n-1}^{1}, z_{n}^{1}\right), d_{2}\left(z_{n}^{2}, z_{n+1}^{2}\right)\right\} \\
d_{3}\left(z_{n}^{3}, z_{n+1}^{3}\right) \leqslant & c \max _{\{} d_{1}\left(z_{n-1}^{1}, z_{n}^{1}\right), d_{2}\left(z_{n-1}^{2}, z_{n}^{2}\right), \\
& \left.d_{3}\left(z_{n-1}^{3}, z_{n}^{3}\right), d_{4}\left(z_{n-1}^{4}, z_{n}^{4}\right)\right\}
\end{aligned}
$$


Using inequality (7), we have,

$$
\begin{aligned}
\mathrm{d}_{4}\left(z_{n}^{4}, z_{n+1}^{4}\right)= & d_{4}\left(A_{3} A_{2} A_{1} A_{4} z_{n-1}^{4}, A_{3} A_{2} A_{1} A_{4} z_{n}^{4}\right) \\
\leqslant & c \max \left\{d_{4}\left(z_{n-1}^{4}, z_{n}^{4}\right), d_{4}\left(z_{n-1}^{4}, A_{3} A_{2} A_{1} A_{4} z_{n-1}^{4}\right)\right. \\
& d_{4}\left(z_{n}^{4}, A_{3} A_{2} A_{1} A_{4} z_{n}^{4}\right), d_{1}\left(A_{4} z_{n-1}^{4}, A_{4} z_{n}^{4}\right), \\
& \left.d_{2}\left(A_{1} A_{4} z_{n-1}^{4}, A_{1} A_{4} z_{n}^{4}\right), d_{3}\left(A_{2} A_{1} A_{4} z_{n-1}^{4}, A_{2} A_{1} A_{4} z_{n}^{4}\right)\right\} \\
d_{4}\left(z_{n}^{4}, z_{n+1}^{4}\right) \leqslant & c \max \left\{d_{4}\left(z_{n-1}^{4}, z_{n}^{4}\right), d_{4}\left(z_{n-1}^{4}, z_{n}^{4}\right), d_{4}\left(z_{n}^{4}, z_{n+1}^{4}\right)\right. \\
& \left.d_{1}\left(z_{n-1}^{1}, z_{n}^{1}\right), d_{2}\left(z_{n}^{2}, z_{n+1}^{2}\right), d_{3}\left(z_{n}^{3}, z_{n+1}^{3}\right)\right\} \\
d_{4}\left(z_{n}^{4}, z_{n+1}^{4}\right) \leqslant & c \max \left\{d_{1}\left(z_{n-1}^{1}, z_{n}^{1}\right), d_{2}\left(z_{n-1}^{2}, z_{n}^{2}\right)\right. \\
& \left.d_{3}\left(z_{n-1}^{3}, z_{n}^{3}\right), d_{4}\left(z_{n-1}^{4}, z_{n}^{4}\right)\right\}
\end{aligned}
$$

Using inequality (4), we have,

$$
\begin{aligned}
d_{1}\left(z_{n}^{1}, z_{n+1}^{1}\right)= & d_{1}\left(A_{4} A_{3} A_{2} A_{1} z_{n-1}^{1}, A_{4} A_{3} A_{2} A_{1} z_{n}^{1}\right) \\
\leqslant & c \max \left\{d_{1}\left(z_{n-1}^{1}, z_{n}^{1}\right), d_{1}\left(z_{n-1}^{1}, A_{4} A_{3} A_{2} A_{1} z_{n-1}^{1}\right),\right. \\
& d_{1}\left(z_{n}^{1}, A_{4} A_{3} A_{2} A_{1} z_{n}^{1}\right), d_{2}\left(A_{1} z_{n-1}^{1}, A_{1} z_{n}^{1}\right), \\
& \left.d_{3}\left(A_{2} A_{1} z_{n-1}^{1}, A_{2} A_{1} z_{n}^{1}\right), d_{4}\left(A_{3} A_{2} A_{1} z_{n-1}^{1}, A_{3} A_{2} A_{1} z_{n}^{1}\right)\right\} \\
d_{1}\left(z_{n}^{1}, z_{n+1}^{1}\right) \leqslant & c \max _{\{} d_{1}\left(z_{n-1}^{1}, z_{n}^{1}\right), d_{1}\left(z_{n-1}^{1}, z_{n}^{1}\right), d_{1}\left(z_{n}^{1}, z_{n+1}^{1}\right), \\
& \left.d_{2}\left(z_{n}^{2}, z_{n+1}^{2}\right), d_{3}\left(z_{n}^{3}, z_{n+1}^{3}\right), d_{4}\left(z_{n}^{4}, z_{n+1}^{4}\right)\right\} \\
d_{1}\left(z_{n}^{1}, z_{n+1}^{1}\right) \leqslant & c \max \left\{d_{1}\left(z_{n-1}^{1}, z_{n}^{1}\right), d_{2}\left(z_{n-1}^{2}, z_{n}^{2}\right),\right. \\
& \left.d_{3}\left(z_{n-1}^{3}, z_{n}^{3}\right), d_{4}\left(z_{n-1}^{4}, z_{n}^{4}\right)\right\}
\end{aligned}
$$

By induction on using inequalities (8), (9), (10) and (11), we have,

$$
\begin{aligned}
\mathrm{d}_{1}\left(z_{\mathfrak{n}}^{1}, z_{\mathrm{n}+1}^{1} \leqslant \leqslant\right. & \mathrm{c}^{\mathrm{n}-1} \max \left\{\mathrm{d}_{1}\left(z_{1}^{1}, z_{2}^{1}\right), \mathrm{d}_{2}\left(z_{1}^{2}, z_{2}^{2}\right),\right. \\
& \left.\mathrm{d}_{3}\left(z_{1}^{3}, z_{2}^{3}\right), \mathrm{d}_{4}\left(z_{1}^{4}, z_{2}^{4}\right)\right\} \\
\mathrm{d}_{2}\left(z_{\mathfrak{n}}^{2}, z_{\mathrm{n}+1}^{2} \leqslant \leqslant\right. & \mathrm{c}^{\mathrm{n}-1} \max \left\{\mathrm{d}_{1}\left(z_{1}^{1}, z_{2}^{1}\right), \mathrm{d}_{2}\left(z_{1}^{2}, z_{2}^{2}\right),\right. \\
& \left.\mathrm{d}_{3}\left(z_{1}^{3}, z_{2}^{3}\right), \mathrm{d}_{4}\left(z_{1}^{4}, z_{2}^{4}\right)\right\} \\
\mathrm{d}_{3}\left(z_{\mathrm{n}}^{3}, z_{\mathrm{n}+1}^{3} \leqslant \leqslant\right. & \mathrm{c}^{\mathrm{n}-1} \max \left\{\mathrm{d}_{1}\left(z_{1}^{1}, z_{2}^{1}\right), \mathrm{d}_{2}\left(z_{1}^{2}, z_{2}^{2}\right),\right. \\
& \left.\mathrm{d}_{3}\left(z_{1}^{3}, z_{2}^{3}\right), \mathrm{d}_{4}\left(z_{1}^{4}, z_{2}^{4}\right)\right\} \\
\mathrm{d}_{4}\left(z_{n}^{4}, z_{\mathrm{n}+1}^{4} \leqslant \leqslant\right. & \mathrm{c}^{\mathrm{n}-1} \max \left\{\mathrm{d}_{1}\left(z_{1}^{1}, z_{2}^{1}\right), \mathrm{d}_{2}\left(z_{1}^{2}, z_{2}^{2}\right),\right. \\
& \left.\mathrm{d}_{3}\left(z_{1}^{3}, z_{2}^{3}\right), \mathrm{d}_{4}\left(z_{1}^{4}, z_{2}^{4}\right)\right\}
\end{aligned}
$$


Since $c<1$, it follows that $\left\{z_{n}^{1}\right\},\left\{z_{n}^{2}\right\},\left\{z_{n}^{3}\right\}$ and $\left\{z_{n}^{4}\right\}$ are Cauchy sequences with limit $\alpha_{1}, \alpha_{2}, \alpha_{3}$ and $\alpha_{4}$ in $Z_{1}, Z_{2}, Z_{3}$ and $Z_{4}$ respectively.

Since $A_{1}, A_{2}$ and $A_{3}$ are continuous, we have,

$$
\begin{aligned}
& \lim _{n \rightarrow \infty} z_{n}^{2}=\lim _{n \rightarrow \infty} A_{1} z_{n}^{1}=A_{1} \alpha_{1}=\alpha_{2} \\
& \lim _{n \rightarrow \infty} z_{n}^{3}=\lim _{n \rightarrow \infty} A_{2} z_{n}^{2}=A_{2} \alpha_{2}=\alpha_{3} \\
& \lim _{n \rightarrow \infty} z_{n}^{4}=\lim _{n \rightarrow \infty} A_{3} z_{n}^{3}=A_{3} \alpha_{3}=\alpha_{4}
\end{aligned}
$$

Using inequality (4), again, we get,

$$
\begin{aligned}
d_{1}\left(A_{4} A_{3} A_{2} A_{1} \alpha_{1}, z_{n}^{1}\right)= & d_{1}\left(A_{4} A_{3} A_{2} A_{1} \alpha_{1}, A_{4} A_{3} A_{2} A_{1} z_{n-1}^{1}\right) \\
\leqslant & c \max \left\{d_{1}\left(\alpha_{1}, z_{n-1}^{1}\right), d_{1}\left(\alpha_{1}, A_{4} A_{3} A_{2} A_{1} \alpha_{1}\right),\right. \\
& d_{1}\left(z_{n-1}^{1}, A_{4} A_{3} A_{2} A_{1} z_{n-1}^{1}\right), d_{2}\left(A_{1} \alpha_{1}, A_{1} z_{n-1}^{1}\right), \\
& \left.d_{3}\left(A_{2} A_{1} \alpha_{1}, A_{2} A_{1} z_{n-1}^{1}\right), d_{4}\left(A_{3} A_{2} A_{1} \alpha_{1}, A_{3} A_{2} A_{1} z_{n-1}^{1}\right)\right\}
\end{aligned}
$$

Since $A_{1}, A_{2}$ and $A_{3}$ are continuous, it follows on letting $n \rightarrow \infty$ that

$$
d_{1}\left(A_{4} A_{3} A_{2} A_{1} \alpha_{1}, \alpha_{1}\right) \leqslant c \max \left\{d_{1}\left(\alpha_{1}, A_{4} A_{3} A_{2} A_{1} \alpha_{1}\right)\right\}
$$

Thus, we have, $A_{4} A_{3} A_{2} A_{1} \alpha_{1}=\alpha_{1}$. Since $c<1$ and $\alpha_{1}$ is the fixed point of $A_{4} A_{3} A_{2} A_{1}$

and

$$
A_{1} A_{4} A_{3} A_{2} \alpha_{2}=A_{1} A_{4} A_{3} A_{2} A_{1} \alpha_{1}=A_{1} \alpha_{1}=\alpha_{2}
$$

and

$$
A_{2} A_{1} A_{4} A_{3} \alpha_{3}=A_{2} A_{1} A_{4} A_{3} A_{2} \alpha_{2}=A_{2} \alpha_{2}=\alpha_{3}
$$$$
A_{3} A_{2} A_{1} A_{4} \alpha_{4}=A_{3} A_{2} A_{1} A_{4} A_{3} \alpha_{3}=A_{3} \alpha_{3}=\alpha_{4}
$$

Hence $\alpha_{2}, \alpha_{3}$ and $\alpha_{4}$ are fixed points of $A_{1} A_{4} A_{3} A_{2}, A_{2} A_{1} A_{4} A_{3}$ and $A_{3} A_{2} A_{1} A_{4}$ respectively.

\subsection{Uniqueness}

Suppose that $A_{4} A_{3} A_{2} A_{1}$ has a second fixed point $\alpha_{1}^{\prime}$. Then, using inequality (4), we have,

$$
\begin{aligned}
d_{1}\left(\alpha_{1}, \alpha_{1}^{\prime}\right)= & d_{1}\left(A_{4} A_{3} A_{2} A_{1} \alpha_{1}, A_{4} A_{3} A_{2} A_{1} \alpha_{1}^{\prime}\right) \\
\leqslant & c \max \left\{d_{1}\left(\alpha_{1}, \alpha_{1}^{\prime}\right), d_{1}\left(\alpha_{1}, A_{4} A_{3} A_{2} A_{1} \alpha_{1}\right),\right. \\
& d_{1}\left(\alpha_{1}^{\prime}, A_{4} A_{3} A_{2} A_{1} \alpha_{1}^{\prime}\right), d_{2}\left(A_{1} \alpha_{1}, A_{1} \alpha_{1}^{\prime}\right), \\
& d_{3}\left(A_{2} A_{1} \alpha_{1}, A_{2} A_{1} \alpha_{1}^{\prime}\right) \\
& \left.d_{4}\left(A_{3} A_{2} A_{1} \alpha_{1}, A_{3} A_{2} A_{1} \alpha_{1}^{\prime}\right)\right\}
\end{aligned}
$$




$$
\begin{aligned}
d_{1}\left(\alpha_{1}, \alpha_{1}^{\prime}\right) \leqslant & c \max \left\{d_{1}\left(\alpha_{1}, \alpha_{1}^{\prime}\right), d_{1}\left(\alpha_{1}, \alpha_{1}\right), d_{1}\left(\alpha_{1}^{\prime}, \alpha_{1}^{\prime}\right),\right. \\
& d_{2}\left(A_{1} \alpha_{1}, A_{1} \alpha_{1}^{\prime}\right), d_{3}\left(A_{2} A_{1} \alpha_{1}, A_{2} A_{1} \alpha_{1}^{\prime}\right), \\
& \left.d_{4}\left(A_{3} A_{2} A_{1} \alpha_{1}, A_{3} A_{2} A_{1} \alpha_{1}^{\prime}\right)\right\} \\
d_{1}\left(\alpha_{1}, \alpha_{1}^{\prime}\right) \leqslant & c \max \left\{d_{2}\left(A_{1} \alpha_{1}, A_{1} \alpha_{1}^{\prime}\right), d_{3}\left(A_{2} A_{1} \alpha_{1}, A_{2} A_{1} \alpha_{1}^{\prime}\right),\right. \\
& \left.d_{4}\left(A_{3} A_{2} A_{1} \alpha_{1}, A_{3} A_{2} A_{1} \alpha_{1}^{\prime}\right)\right\}
\end{aligned}
$$

Using inequality (5), we have

$$
\begin{aligned}
& d_{2}\left(A_{1} \alpha_{1}, A_{1} \alpha_{1}^{\prime}\right)=d_{2}\left(A_{1} A_{4} A_{3} A_{2} A_{1} \alpha_{1}, A_{1} A_{4} A_{3} A_{2} A_{1} \alpha_{1}^{\prime}\right) \\
& \leqslant c \max \left\{d_{2}\left(A_{1} \alpha_{1}, A_{1} \alpha_{1}^{\prime}\right)\right. \text {, } \\
& \mathrm{d}_{2}\left(A_{1} \alpha_{1}, A_{1} A_{4} A_{3} A_{2} A_{1} \alpha_{1}\right) \text {, } \\
& \mathrm{d}_{2}\left(A_{1} \alpha_{1}^{\prime}, A_{1} A_{4} A_{3} A_{2} A_{1} \alpha_{1}^{\prime}\right) \text {, } \\
& \mathrm{d}_{3}\left(A_{2} A_{1} \alpha_{1}, A_{2} A_{1} \alpha_{1}^{\prime}\right) \text {, } \\
& \mathrm{d}_{4}\left(A_{3} A_{2} A_{1} \alpha_{1}, A_{3} A_{2} A_{1} \alpha_{1}^{\prime}\right) \text {, } \\
& \left.\mathrm{d}_{4}\left(A_{4} A_{3} A_{2} A_{1} \alpha_{1}, A_{4} A_{3} A_{2} A_{1} \alpha_{1}^{\prime}\right)\right\} \\
& d_{2}\left(A_{1} \alpha_{1}, A_{1} \alpha_{1}^{\prime}\right) \leqslant c \max \left\{d_{2}\left(A_{1} \alpha_{1}, A_{1} \alpha_{1}^{\prime}\right), d_{2}\left(A_{1} \alpha_{1}, A_{1} \alpha_{1}^{\prime}\right),\right. \\
& \mathrm{d}_{1}\left(A_{1} \alpha_{1}^{\prime}, A_{1} \alpha_{1}^{\prime}\right) \text {, } \\
& d_{3}\left(A_{2} A_{1} \alpha_{1}, A_{2} A_{1} \alpha_{1}^{\prime}\right) \text {, } \\
& \mathrm{d}_{4}\left(A_{3} A_{2} A_{1} \alpha_{1}, A_{3} A_{2} A_{1} \alpha_{1}^{\prime}\right) \text {, } \\
& \left.d_{1}\left(A_{4} A_{3} A_{2} A_{1} \alpha_{1}, A_{4} A_{3} A_{2} A_{1} \alpha_{1}^{\prime}\right)\right\} \\
& d_{2}\left(A_{1} \alpha_{1}, A_{1} \alpha_{1}^{\prime}\right) \leqslant c \max \left\{d_{2}\left(A_{1} \alpha_{1}, A_{1} \alpha_{1}^{\prime}\right), d_{3}\left(A_{2} A_{1} \alpha_{1}, A_{2} A_{1} \alpha_{1}^{\prime}\right)\right. \text {, } \\
& \left.d_{4}\left(A_{3} A_{2} A_{1} \alpha_{1}, A_{3} A_{2} A_{1} \alpha_{1}^{\prime}\right), d_{1}\left(\alpha_{1}, \alpha_{1}^{\prime}\right)\right\} \\
& d_{2}\left(A_{1} \alpha_{1}, A_{1} \alpha_{1}^{\prime}\right) \leqslant c \max \left\{d_{3}\left(A_{2} A_{1} \alpha_{1}, A_{2} A_{1} \alpha_{1}^{\prime}\right)\right. \text {, } \\
& \left.d_{4}\left(A_{3} A_{2} A_{1} \alpha_{1}, A_{3} A_{2} A_{1} \alpha_{1}^{\prime}\right), d_{1}\left(\alpha_{1}, \alpha_{1}^{\prime}\right)\right\}
\end{aligned}
$$

Now, we have

$$
\begin{aligned}
d_{2}\left(A_{1} \alpha_{1}, A_{1} \alpha_{1}^{\prime}\right) \leqslant & c \max \left\{d_{3}\left(A_{2} A_{1} \alpha_{1}, A_{2} A_{1} \alpha_{1}^{\prime}\right)\right. \\
& d_{4}\left(A_{3} A_{2} A_{1} \alpha_{1}, A_{3} A_{2} A_{1} \alpha_{1}^{\prime}\right), \\
& c d_{2}\left(A_{1} \alpha_{1}, A_{1} \alpha_{1}^{\prime}\right), d_{3}\left(A_{2} A_{1} \alpha_{1}, A_{2} A_{1} \alpha_{1}^{\prime}\right), \\
& \left.c d_{4}\left(A_{3} A_{2} A_{1} \alpha_{1}, A_{3} A_{2} A_{1} \alpha_{1}^{\prime}\right)\right\} \\
d_{2}\left(A_{1} \alpha_{1}, A_{1} \alpha_{1}^{\prime}\right) \leqslant & c \max \left\{d_{3}\left(A_{2} A_{1} \alpha_{1}, A_{2} A_{1} \alpha_{1}^{\prime}\right)\right. \\
& \left.d_{4}\left(A_{3} A_{2} A_{1} \alpha_{1}, A_{3} A_{2} A_{1} \alpha_{1}^{\prime}\right)\right\}
\end{aligned}
$$


Similarly on using inequality (6), we get

$$
\begin{aligned}
d_{3}\left(A_{2} A_{1} \alpha_{1}, A_{2} A_{1} \alpha_{1}^{\prime}\right) \leqslant & c \max \left\{d_{3}\left(A_{2} A_{1} \alpha_{1}, A_{2} A_{1} \alpha_{1}^{\prime}\right),\right. \\
& d_{3}\left(A_{2} A_{1} \alpha_{1}, A_{2} A_{1} A_{4} A_{3} A_{2} A_{1} \alpha_{1}\right), \\
& d_{3}\left(A_{2} A_{1} \alpha_{1}^{\prime}, A_{2} A_{1} A_{4} A_{3} A_{2} A_{1} \alpha_{1}^{\prime}\right), \\
& d_{4}\left(A_{3} A_{2} A_{1} \alpha_{1}, A_{3} A_{2} A_{1} \alpha_{1}^{\prime}\right), \\
& d_{1}\left(A_{4} A_{3} A_{2} A_{1} \alpha_{1}, A_{4} A_{3} A_{2} A_{1} \alpha_{1}^{\prime}\right), \\
& \left.d_{2}\left(A_{1} A_{4} A_{3} A_{2} A_{1} \alpha_{1}, A_{1} A_{4} A_{3} A_{2} A_{1} \alpha_{1}^{\prime}\right)\right\} \\
d_{3}\left(A_{2} A_{1} \alpha_{1}, A_{2} A_{1} \alpha_{1}^{\prime}\right) \leqslant & c \max _{3} d_{3}\left(A_{2} A_{1} \alpha_{1}, A_{2} A_{1} \alpha_{1}^{\prime}\right), \\
& d_{3}\left(A_{2} A_{1} \alpha_{1}, A_{2} A_{1} \alpha_{1}^{\prime}\right), \\
& d_{3}\left(A_{2} A_{1} \alpha_{1}^{\prime}, A_{2} A_{1} \alpha_{1}^{\prime}\right), \\
& d_{4}\left(A_{3} A_{2} A_{1} \alpha_{1}, A_{3} A_{2} A_{1} \alpha_{1}^{\prime}\right), \\
& \left.d_{1}\left(\alpha_{1}, \alpha_{1}^{\prime}\right), d_{2}\left(A_{1} \alpha_{1}, A_{1} \alpha_{1}^{\prime}\right)\right\} \\
d_{3}\left(A_{2} A_{1} \alpha_{1}, A_{2} A_{1} \alpha_{1}^{\prime}\right) \leqslant & c \max _{\{} d_{4}\left(A_{3} A_{2} A_{1} \alpha_{1}, A_{3} A_{2} A_{1} \alpha_{1}^{\prime}\right), \\
& \left.d_{1}\left(\alpha_{1}, \alpha_{1}^{\prime}\right), d_{2}\left(A_{1} \alpha_{1}, A_{1} \alpha_{1}^{\prime}\right)\right\} \\
d_{3}\left(A_{2} A_{1} \alpha_{1}, A_{2} A_{1} \alpha_{1}^{\prime}\right) \leqslant & c \max _{2} d_{4}\left(A_{3} A_{2} A_{1} \alpha_{1}, A_{3} A_{2} A_{1} \alpha_{1}^{\prime}\right), \\
& c d_{2}\left(A_{1} \alpha_{1}, A_{1} \alpha_{1}^{\prime}\right), c d_{3}\left(A_{2} A_{1} \alpha_{1}, A_{2} A_{1} \alpha_{1}^{\prime}\right) \\
& \left.c d_{4}\left(A_{3} A_{2} A_{1} \alpha_{1}, A_{3} A_{2} A_{1} \alpha_{1}^{\prime}\right)\right\}
\end{aligned}
$$

Using inequality (13) and (14), we have

$$
d_{3}\left(A_{2} A_{1} \alpha_{1}, A_{2} A_{1} \alpha_{1}^{\prime}\right) \leqslant c \max \left\{d_{4}\left(A_{3} A_{2} A_{1} \alpha_{1}, A_{3} A_{2} A_{1} \alpha_{1}^{\prime}\right)\right\}
$$

Similarly on using inequality (7), (13) and (15), we have,

$$
\left.d_{4}\left(A_{3} A_{2} A_{1} \alpha_{1}, A_{3} A_{2} A_{1} \alpha_{1}^{\prime}\right)\right) \leqslant c d_{1}\left(\alpha_{1}, \alpha_{1}^{\prime}\right)
$$

Using inequality (12), (13), (15) and (16), we have

$$
\begin{aligned}
d_{1}\left(\alpha_{1}, \alpha_{1}^{\prime}\right) & \leqslant c d_{2}\left(A_{1} \alpha_{1}, A_{1} \alpha_{1}^{\prime}\right) \\
& \leqslant c^{2} d_{3}\left(A_{2} A_{1} \alpha_{1}, A_{2} A_{1} \alpha_{1}^{\prime}\right) \\
& \leqslant c^{3} d_{4}\left(A_{3} A_{2} A_{1} \alpha_{1}, A_{3} A_{2} A_{1} \alpha_{1}^{\prime}\right) \\
& \leqslant c^{4} d_{1}\left(\alpha_{1}, \alpha_{1}^{\prime}\right)
\end{aligned}
$$

Now we have

$$
\mathrm{d}_{1}\left(\alpha_{1}, \alpha_{1}^{\prime}\right) \leqslant \mathrm{c}^{4} \mathrm{~d}_{1}\left(\alpha_{1}, \alpha_{1}^{\prime}\right)
$$


Since $0 \leqslant c<1$, we have

$$
\mathrm{d}_{1}\left(\alpha_{1}, \alpha_{1}^{\prime}\right)=0
$$

$\Rightarrow \quad \alpha_{1}=\alpha_{1}^{\prime}$, proving the uniqueness of $\alpha_{1}$.

We can similarly prove that $A_{1} A_{4} A_{3} A_{2}$ has a unique fixed point $d_{2} \in Z_{2}$ and $A_{2} A_{1} A_{4} A_{3}$ has a unique fixed point $\alpha_{3} \in Z$ and $A_{3} A_{2} A_{1} A_{4}$ has unique fixed point $\alpha_{4} \in Z_{4}$.

Now, in support of our result, we give some examples.

Example 1 Let suppose $\mathrm{X}=[0,1], \mathrm{Y}=[1,2], \mathrm{Z}=[2,3]$ and $\mathrm{L}=[3,4]$ be complete metric spaces with usual metric. If $\mathrm{T}:[0,1] \rightarrow[1,2], \mathrm{S}:[1,2] \rightarrow[2,3]$ and $\mathrm{R}:[2,3] \rightarrow[3,4]$ are continuous mappings and $\mathrm{U}:[3,4] \rightarrow[0,1]$ is a mapping satisfying given conditions (in Theorem 2.1), where

$$
\begin{aligned}
& T(x)=\left\{\begin{array}{ll}
1, & \text { if } \quad 0 \leq x \leq \frac{3}{4} \\
\frac{4}{3} x, & \text { if } \quad \frac{3}{4}<x \leq 1
\end{array}, \quad S(y)=\left\{\begin{array}{lll}
2, & \text { if } \quad 1 \leq y \leq \frac{3}{2} \\
\frac{4}{3} y, & \text { if } \quad \frac{3}{2}<y \leq 2
\end{array}\right.\right. \\
& R(z)=\left\{\begin{array}{ll}
3, & \text { if } 2 \leq z \leq \frac{5}{2} \\
\frac{6}{5} z, & \text { if } \frac{5}{2}<z \leq 3
\end{array}, \quad U(u)= \begin{cases}1, & \text { if } 3 \leq u \leq \frac{7}{2} \\
\frac{3}{5}, & \text { if } \frac{7}{2}<u \leq 4\end{cases} \right.
\end{aligned}
$$

then URST has fixed point 1 such that URST(1)=1, TURS has fixed point 4/3 such that TURS $(4 / 3)=4 / 3$, STUR has fixed point 2 such that STUR $(2)=2$ and RSTU has fixed point 3 such that RSTU(3) $=3$. Also $\mathrm{T}(1)=4 / 3, \mathrm{~S}(4 / 3)=2$, $\mathrm{R}(2)=3$ and $\mathrm{U}(3)=1$.

Remark 1 Below we give an example which satisfies all the condition of Theorem 2.1 but does not satisfies the condition of Theorem 1.1.

Example 2 Let $\mathrm{X}=[0,1], \mathrm{Y}=[1,2], \mathrm{Z}=[2,3]$ and $\mathrm{L}=[3,4]$ be complete metric space with usual metric. If $\mathrm{T}:[0,1] \rightarrow[1,2], \mathrm{S}:[1,2] \rightarrow[2,3]$ and $\mathrm{R}:[2,3] \rightarrow[3,4]$ are continuous mappings and $\mathrm{U}:[3,4] \rightarrow[0,1]$ is a mapping satisfying given conditions (in Theorem 2.1), where

$$
\begin{aligned}
& \mathrm{T}(\mathrm{x})=\left\{\begin{array}{ll}
1, & \text { if } 0 \leq x \leq \frac{1}{4} \\
2 x+\frac{1}{2}, & \text { if } \frac{1}{4} \leq x \leq \frac{3}{4}, \\
2, & \text { if } \frac{3}{4} \leq x \leq 1
\end{array} \quad \mathrm{~S}(\mathrm{y})=\left\{\begin{array}{lll}
2, & \text { if } 1 \leq y \leq \frac{5}{4} \\
\frac{4}{5} y+1, & \text { if } \frac{5}{4} \leq \mathrm{y} \leq \frac{7}{4} \\
2.4, & \text { if } \quad \frac{7}{4} \leq \mathrm{y} \leq 2
\end{array}\right.\right. \\
& \mathrm{R}(z)=\left\{\begin{array}{ll}
3, & \text { if } 2 \leq z \leq \frac{9}{4} \\
z+\frac{3}{4}, & \text { if } \frac{9}{4} \leq z \leq \frac{11}{4}, \\
3.5, & \text { if } \frac{11}{4} \leq z \leq 3
\end{array} \quad \mathrm{u}(\mathrm{u})=\left\{\begin{array}{lll}
0, & \text { if } & 3 \leq \mathrm{u} \leq \frac{7}{2} \\
\frac{2}{7} u-1, & \text { if } \quad \frac{7}{2} \leq \mathrm{u} \leq 4
\end{array}\right.\right.
\end{aligned}
$$


then URST has fixed point 0 such that URST $(0)=0$, TURS has fixed point 1 such that TURS $(1)=1$, STUR has fixed point 2 such that $\operatorname{STUR}(2)=2$ and RSTU has fixed point 3 such that RSTU( 3$)=3$. Also $\mathrm{T}(0)=1, \mathrm{~S}(1)=2, \mathrm{R}(2)=3$ and $\mathrm{U}(3)=0$.

Example 3 Let suppose $\mathrm{X}=[0,2], \mathrm{Y}=[1,5], \mathrm{Z}=[0,10]$ and $\mathrm{L}=[1,12]$ be complete metric spaces with usual metric. If $\mathrm{T}:[0,2] \rightarrow[1,5], \mathrm{S}:[1,5] \rightarrow[0,10]$ and $\mathrm{R}:[0,10] \rightarrow[1,12]$ are continuous mappings and $\mathrm{U}:[1,12] \rightarrow[0,2]$ is a mapping satisfying given conditions (in Theorem 2.1), where

$$
\begin{array}{rr}
\mathrm{T}(\mathrm{x})=[1+x, 2], & \mathrm{S}(\mathrm{y})=[2 \mathrm{y}+1,5] \\
\mathrm{R}(z)=[1+z, 10], & \mathrm{U}(\mathrm{u})=\left\{\begin{array}{lll}
{\left[\frac{\mathrm{u}}{6}, 5\right]} & \text { if } & 1 \leq z \leq 6 \\
{\left[1, \frac{\mathrm{u}}{6}\right]} & \text { if } & 6<z \leq 12
\end{array}\right.
\end{array}
$$

then URST has fixed point 1 such that URST(1) =1, TURS has fixed point 2 such that TURS(2) $=2$, STUR has fixed point 5 such that STUR(5) $=5$ and RSTU has fixed point 6 such that $\operatorname{RSTU}(6)=6$. Also $\mathrm{T}(1)=2, \mathrm{~S}(2)=5$, $\mathrm{R}(5)=6$ and $\mathrm{U}(6)=1$.

Example 4 Let suppose $\mathrm{X}=[0,3], \mathrm{Y}=[1,4], \mathrm{Z}=[4,7]$ and $\mathrm{L}=[3,10]$ be complete metric spaces with usual metric. If $\mathrm{T}:[0,3] \rightarrow[1,4], \mathrm{S}:[1,4] \rightarrow[4,7]$ and $\mathrm{R}:[4,7] \rightarrow[3,10]$ be a continuous mappings and $\mathrm{U}:[3,10] \rightarrow[0,3]$ be a mapping satisfying given conditions (in Theorem 2.1), where

$$
\begin{gathered}
\mathrm{T}(\mathrm{x})=1+x, \quad \mathrm{y})=\mathrm{y}+2, \\
\mathrm{R}(\mathrm{z})=z+3, \quad \mathrm{u}(\mathrm{u})=\left\{\begin{array}{lll}
\frac{\mathrm{u}}{7} & \text { if } & 3 \leq z \leq 5 \\
\frac{\mathrm{u}+1}{8} & \text { if } & 5<z \leq 10
\end{array}\right.
\end{gathered}
$$

then URST has fixed point 1 such that URST(1)=1, TURS has fixed point 2 such that TURS $(2)=2$, STUR has fixed point 4 such that STUR(4) $=4$ and RSTU has fixed point 7 such that $\operatorname{RSTU}(7)=7$. Also $\mathrm{T}(1)=2, \mathrm{~S}(2)=4$, $\mathrm{R}(5)=7$ and $\mathrm{U}(7)=1$.

\section{References}

[1] Z. K. Ansari, M. Sharma, A. Garg, Related fixed points theorems on three metric spaces, Int. J. Contemp. Math. Sci., 5 (42) (2010), 2059-2064. 
[2] B. Fisher, Related fixed points on two metric spaces, Math. Sem. Notes, Kobe Univ., 10 1982, 17-26.

[3] B. Fisher, K. P. R. Rao, A related fixed point theorem on three metric spaces, Hacet. J. Math. Stat., 32 (2) (2007), 143-146.

[4] R. K. Jain, H. K. Sahu, B. Fisher, Related fixed point theorems for three metric spaces, Novi Sad J. Math., 26 (1) (1996), 11-17.

[5] L. Kikina, K. Kikina, A related fixed point theorem on four metric spaces, Int. J. Math. Anal., 3 (32) (2009), 1559-1568.

[6] K. Kikina, L. Kikina, Generalized fixed point theorem in three metric spaces, Int. J. Math. Anal., 4 (40) (2010), 1995-2004.

[7] R. K. Namdeo, B. Fisher, A related fixed point theorem for three pairs of mappings on three metric spaces, Thai J . Math., 7 (1) (2009), 129-135.

[8] V. Gupta, Fixed point theorems for pair of mappings on three metric spaces, Int. J. Adv. Appl. Sci., 3 (5) (2012), 2733-2738. 